\title{
Brillouin light scattering anomalies and new phase transition in $\mathrm{Cs} 5 \mathrm{H} 3(\mathrm{SO} 4) 4$ crystals
}

\author{
Authors: S.G. Lushnikov, V. Hugo Schmidt, L.A. \\ Shuvalov, and V.V. Dolbinina
}

NOTICE: this is the author's version of a work that was accepted for publication in Solid State Communications. Changes resulting from the publishing process, such as peer review, editing, corrections, structural formatting, and other quality control mechanisms may not be reflected in this document. Changes may have been made to this work since it was submitted for publication. A definitive version was subsequently published in Solid State Communications [VOL\# 113 (February 2000)] DOI\# 10.1016/S0038-1098(99)00552-9

S.G. Lushnikov, V.H. Schmidt, L.A. Shuvalov, and V.V. Dolbinina, "Brillouin light scattering anomalies and new phase transition in $\mathrm{Cs} 5 \mathrm{H} 3(\mathrm{SO} 4) 4$ crystals," Solid State Communications 113, 639-642 (February 2000). http://dx.doi.org/10.1016/S0038-1098(99)00552-9

Made available through Montana State University's ScholarWorks scholarworks. montana.edu 


\title{
Brillouin light scattering anomalies and new phase transition in $\mathrm{Cs}_{5} \mathrm{H}_{3}\left(\mathrm{SO}_{4}\right)_{4}$ crystals
}

\author{
S.G. Lushnikov, ${ }^{\mathrm{a}}$, V.H. Schmidt ${ }^{\mathrm{b}}$, L.A. Shuvalov ${ }^{\mathrm{c}}$, V.V. Dolbinina ${ }^{\mathrm{c}}$ \\ ${ }^{a}$ A.F.Ioffe Physical Technical Institute, Russian Academy of Sciences, 194021, St. Petersburg, Russian Federation \\ ${ }^{\mathrm{b}}$ Physics Department, Montana State University, Bozeman, MT 59717, USA \\ ${ }^{\mathrm{c}}$ Institute of Crystallography RAS, Moscow, Russian Federation
}

Received 4 November 1999; accepted 19 November 1999 by E.L. Ivchenko

\begin{abstract}
The behavior of hypersonic longitudinal acoustic phonons at temperatures ranging from 290 to $370 \mathrm{~K}$ in a crystal of $\mathrm{Cs}_{5} \mathrm{H}_{3}\left(\mathrm{SO}_{4}\right)_{4} \cdot n \mathrm{H}_{2} \mathrm{O}$ was studied by Brillouin light scattering. Anomalies in the temperature dependences of the frequency shift and spectral width of the Brillouin components in the vicinity of $T \approx 360 \mathrm{~K}$ were observed. We found a "point of isotropization" near $360 \mathrm{~K}$ where $C_{11} \approx C_{33} \approx 2.6 \times 10^{10} \mathrm{~N} / \mathrm{m}^{2}$. We attribute it to an isostructural phase transition from $P 6_{3} / m m c \Leftrightarrow P 6_{3} / m m c$ where the apparent acoustic symmetry changes from hexagonal to cubic. Possible models for the phase transition have been discussed. (c) 2000 Published by Elsevier Science Ltd. All rights reserved.
\end{abstract}

Keywords: D. Acoustic properties; D. Phase transitions; D. Phonons

\section{Introduction}

This work describes studies of Brillouin light scattering in the $\mathrm{Cs}_{5} \mathrm{H}_{3}\left(\mathrm{SO}_{4}\right)_{4} \cdot n \mathrm{H}_{2} \mathrm{O}$-(PCTS) crystal. This compound is a representative of superprotonic crystals with a dynamically disordered network of hydrogen bonds in the high-temperature phase. As temperature decreases, the dynamic disorder in this crystal gives way to the static disorder that arises on "freezing" of the orientation and position disorder of the network of hydrogen bonds [1,2]. The PCTS crystal exhibits two features unique to this crystal. They are as follows:

1. As temperature decreases and the crystal passes from the superionic to the proton glass state, dynamic and static disordering are realized consecutively.

2. The protonic glass state results from freezing of the disordered network of acid hydrogen bonds rather than from the competition between the anti- and ferroelectric ordering characteristic of the RADP system.

These features make the PCTS compound a unique object for the studies of partially disordered crystals. The

\footnotetext{
* Corresponding author. Fax: +7-812-515-67-47.

E-mail address: sergey.lushinkov@shuvpop.ioffe.rssi.ru (S.G. Lushnikov).
}

properties of PCTS crystals have been widely studied. For instance, their structure was analyzed by X-ray diffraction $[1,3]$, the conductivity mechanism was studied by dielectric measurements [1,4], neutron scattering [5] and NMR spectroscopy [6], and the vibration spectrum was investigated by IR, Raman, Brillouin and neutron scattering in a wide temperature range [7-10]. Nevertheless, the sequence of phase transitions is still unclear. It has been found that at room temperature the symmetry is $P 6_{3} / \mathrm{mmc}$ and remains the same down to helium temperatures $[1,3]$. There is a more complicated situation with the high-temperature region: with decreasing temperature the crystal passes from the superprotonic phase with the supposed space group $P 6 / \mathrm{mmm}$ at $T=414 \mathrm{~K}$ into the phase with the $P 6_{3} / \mathrm{mmc}$ symmetry. At the same time, the studies of the dynamics of the crystalline lattice of PCTS by Raman scattering have revealed specifi features in the behavior of scattering spectra in the vicinity of $360 \mathrm{~K}[7,9]$ consistent with the anomalies in the temperature dependences of the real part of the dielectric constant $\epsilon^{\prime}$ [4] and spin-spin proton relaxation time $T_{2}$ [6]. Thus there is good reason to believe that at $T=$ $360 \mathrm{~K}$ a phase transition takes place in the PCTS crystal. Large spontaneous deformations leading to a poorer optical quality of the crystals in the vicinity of $360 \mathrm{~K}$ [7], make the studies of acoustic properties of PCTS especially attractive. 


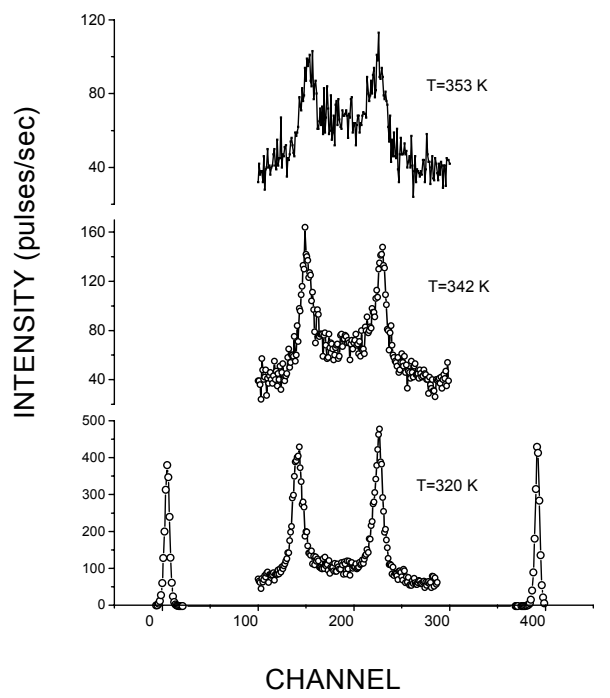

Fig. 1. Three Brillouin spectra at different temperatures are shown to illustrate the experimental conditions to measure three values: the frequency shift, the linewidth at half maximum, and the intensity of the spectra.

Note that when PCTS is cooled again to below $360 \mathrm{~K}$, its optical quality improves.

The goal of this work was to study the behavior of longitudinal hypersonic acoustic phonons in the temperature range of $295-360 \mathrm{~K}$.

\section{Experimental details}

The PCTS crystals were grown by slow evaporation from water solution at room temperature. For the experiments, samples in the form of parallelepipeds were cut so that the hexagonal axis (which lies along a natural crystal rib) was perpendicular to the basal plane. The crystal orientation was determined by a polarizing microscope. The sample surfaces were polished to optical quality. The light source was a single-mode $\mathrm{Ar}^{+}$Spectra-Physics laser with $\lambda=$ $514.5 \mathrm{~nm}$. The scattered light was analyzed by a five-pas piezoscanning Fabry-Perot interferometer. The laser power at the sample was not higher than $50 \mathrm{~mW}$. To control the temperature, a Lake Shore PT-103 calibrated platinum resistance thermometer was used. The error in measuring and determining temperatures was $\pm 0.5 \mathrm{~K}$. The sample was heated from 295 up to $370 \mathrm{~K}$ in a stepwise fashion and the data were collected automatically. The Brillouin spectra were obtained in the backscattering geometry with the different free spectral ranges (FSR) of 12, 13.5, and $32 \mathrm{GHz}$. The examples of experimental spectra for some temperatures are shown in Fig. 1.

The velocity $(V)$ and damping $(\alpha)$ of hypersonic acoustic phonons are related to the frequency shift $(\nu)$ and spectral width (half-width at half maximum) $(\delta)$ of the Brillouin components in our scattering geometry by

$\nu=2 n V\left(\nu_{0} / c\right)$,

$\alpha=2 \pi \delta / V$,

where $n$ is the refractive index, $\nu_{0}$ is the frequency of the incident light and $c$ is the speed of light. To describe the experimental spectra, the Lorentz function was used because the condition $\alpha / \mathbf{q} \ll 1$ (where $\mathbf{q}$ is the wave vector of longitudinal acoustic (LA) phonons) was fulfilled The experimental spectra were processed using the PeakFit (Jandel Scientific software, which allowed a highly precise determination of the positions and widths of the Brillouin components. Unfortunately, we could not obtain Brillouin components in the scattering spectra at $T>360 \mathrm{~K}$ because at $360 \mathrm{~K}$ the contribution of elastic (Raleigh) scattering sharply grew and the background increased while the intensity of the Brillouin components decreased (see Fig. 1).

\section{Experimental results and discussion}

In this work, LA phonons with $\mathbf{q} \|[001]$ and $\mathbf{q} \|[100]$ were studied. In the PCTS crystals belonging to the hexagonal system, the velocity of the LA phonon propagating in the [001] direction is given by $\rho V^{2}=C_{33}$, where $\rho$ is the crystal density and $C_{33}$ is the elastic tensor component. In the basal plane of PCTS all propagation directions of the LA phonon are equivalent, so $\rho V^{2}=C_{11}$. According to Eq. (1), the Brillouin shifts $\nu$ for the LA phonons with $\mathbf{q} \|[001]$ and $\mathbf{q} \|[100]$ are determined by $C_{33}$ and $C_{11}$, respectively. At room temperature, $C_{11}=(2.77 \pm 0.01) \times 10^{10} \mathrm{~N} / \mathrm{m}^{2}$ and $C_{33}=(2.69 \pm 0.01) \times 10^{10} \mathrm{~N} / \mathrm{m}^{2}$. Thus, the temperature dependences of the Brillouin shift for the LA phonons with $\mathbf{q} \|[001]$ and $\mathbf{q} \|[100]$ (Fig. 1) provide the changes in $C_{33}$ and $C_{11}$ as temperature is varied.

Let us consider the temperature dependences shown in Fig. 2. It is clearly seen that in the temperature range $295-330 \mathrm{~K}, C_{11}$ and $C_{33}$ vary in nearly the same fashion (curves in Fig. 2 are almost parallel). As temperature is increased, the elastic constants under consideration exhibit strongly differing behaviors, i.e. $C_{11}$ sharply decreases and departs from the linear dependence, while $C_{33}$ behaves as before. Softening of $C_{11}$ continues up to $T \approx 355 \mathrm{~K}$, where $C_{11} \approx C_{33} \approx 2.6 \times 10^{10} \mathrm{~N} / \mathrm{m}^{2}$. Thus in the vicinity of the supposed phase transition temperature $\left(T_{2} \approx 360 \mathrm{~K}\right)$, the PCTS crystal becomes "cubic" or "isotropic" from the point of view of the acoustic symmetry.

It is well known that fourth-rank tensors such as the elastic modulus tensor are described by characteristic surfaces formed by the components of the relevant tensor. For the elastic modulus tensor the characteristic surfaces are related to the phase velocity surfaces of acoustic phonons with corresponding acoustic symmetry [11]. For the PCTS crystal a decrease in $C_{11}$ down to the value of $C_{33}$ means a change in the acoustic symmetry from hexagonal to cubic. It should be borne in mind 


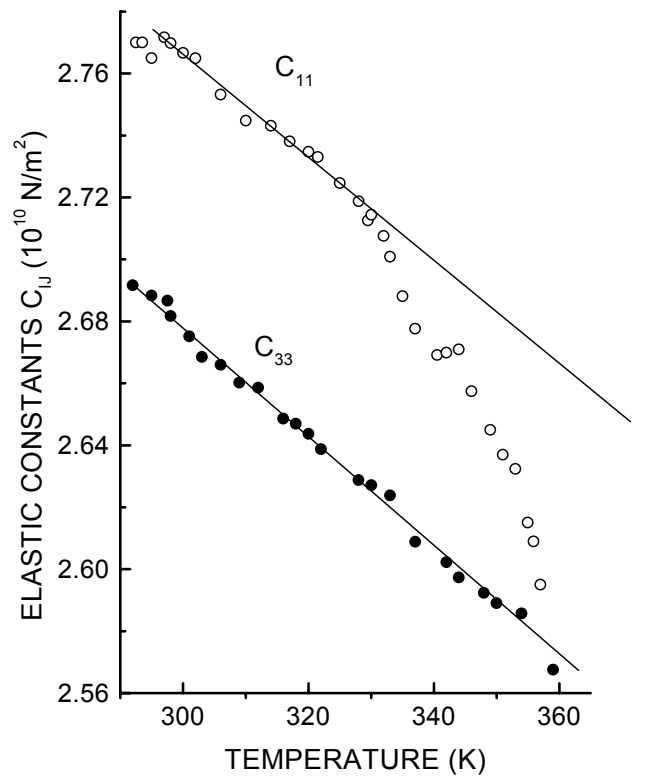

Fig. 2. Temperature dependences of elastic moduli $C_{33}$ and $C_{11}$.

that there is no direct correspondence between the acoustic and crystallographic symmetry. According to Hermann's theorem, they do not coincide in the general case [12].

The use of the term cubic is only partly justifie here because the elastic tensor for hexagonal crystals includes the $C_{13}$ component (which is absent in the cubic crystallographic system) whose temperature behavior in PCTS has not been studied yet.

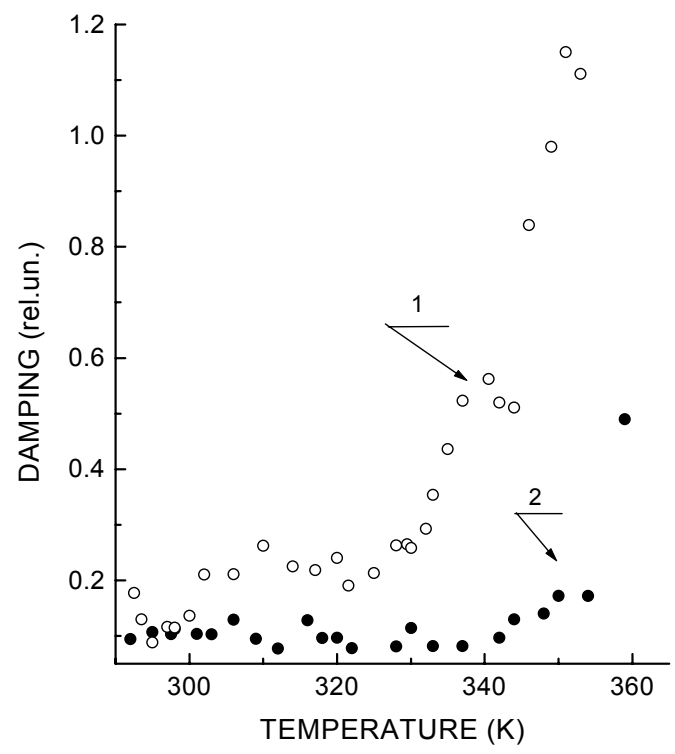

Fig. 3. Temperature dependences the relative changes of the damping of the longitudinal hypersonic acoustic phonons propagating in the basal plane and along the six-fold axis: curves 1 and 2, respectively.
The anomaly in the temperature dependence of $C_{11}$ must have a corresponding anomaly in the acoustic phonon damping. Therefore, by studying the behavior of the spectral width $\delta$ of the Brillouin component with increasing temperature, we obtain information about damping $\alpha$ of the acoustic phonon in the Brillouin scattering experiments through Eq. (2), which establishes the relation between these two values. Thus, the temperature dependences presented in Fig. 3 demonstrate changes in damping of the longitudinal hypersonic elastic waves in the PCTS crystal. At room temperature, the dampings of the LA phonons propagating along the hexagonal axis and in the basal plane (Fig. 3, curves 2 and 1, respectively) are approximately the same. As temperature is increased, these temperature dependences differ markedly, but both exhibit pronounced anomalies in the vicinity of $360 \mathrm{~K}$. A further temperature increase leads to the broadening of the Brillouin component by a factor of 2.5 compared with the initial value (Fig. 3). This damping temperature dependence agrees well with the observed anomaly in the temperature dependence of $C_{11}$ (Figs.1-3). Thus, the behavior of LA phonons confirm the existence of a structural phase transition in the vicinity of $360 \mathrm{~K}$.

The temperature dependence of the real part of the dielectric constant $\epsilon^{\prime}$ in PCTS has a slight inflectio in the vicinity of $T_{2}$ [4]. By discussing the appearance of new modes in the Raman scattering spectra in the temperature region from 360 to $414 \mathrm{~K}$ and analyzing them in the framework of the group-theory approach, the authors of Ref. [7] suggested two explanations for the observed anomalies. According to the firs explanation, on heating from room temperature a transition into an intermediate phase with symmetry below $P 6_{3} / m m c$ occurs. In this case, the selection rules allow the appearance of some additional modes in the Raman spectra. In the second explanation, an isostructural phase transition is realized. However, the increase in the acoustic symmetry of the PCTS crystal from hexagonal to cubic shows that the phase transition model involving a decrease in symmetry is not correct. We attribute the observed anomalies in the Brillouin and Raman light scattering experiments in the vicinity of $360 \mathrm{~K}$ to an isostructural phase transition from $P 6_{3} / m m c \Leftrightarrow P 6_{3} / m m c$. In this transition the space symmetry of the crystal remains unchanged, but the $\mathrm{SO}_{4}$ ions in the $4 \mathrm{e}$ position at vertical ribs of the elementary cell lose stability. The local symmetry $C_{3 v}$ in this case will be realized dynamically. Thus in the case of isostructural phase transition the new modes that appear in the Raman spectra are due to the orientational disorder of sulfate ions [7]. Further investigations of the phase transition at $360 \mathrm{~K}$, by X-ray or neutron scattering, are needed.

\section{Acknowledgements}

This work was supported in part by RFBR Grants 99-0218316 and 96-02-17754 and NSF Grant DMR-9805272. 


\section{References}

[1] A.I. Baranov, O.A. Kabanov, B.V. Merinov, L.A. Shuvalov, Ferroelectrics 127 (1992) 257.

[2] A.I. Baranov, O.A. Kabanov, L.A. Shuvalov, JETP Letters 58 (1993) 548.

[3] B.V. Merinov, A.I. Baranov, L.A. Shuvalov, J. Schneider, H. Schulz, Solid State Ionics 69 (1994) 153.

[4] O.A. Kabanov, Ph.D. thesis, Institute of Crystallography, Moscow, 1993, p. 149.

[5] A.V. Belushkin, C.J. Carlile, L.A. Shuvalov, Ferroelectrics 167 (1995) 21.
[6] A.M. Fajdiga-Bulat, G. Lahajnar, J. Dolinsek, J. Slak, et al., Solid State Ionics 77 (1995) 101.

[7] Yu.I. Yuzyuk, V.P. Dimitriev, V.V. Loshkarev, L.M. Rabkin, L.A. Shuvalov, Ferroelectrics 167 (1995) 53.

[8] Yu.I. Yuzyuk, V.P. Dmitriev, L.M. Rabkin, F. Smutny, et al., Journal of Physics: Condensed Matter 8 (1996) 3965.

[9] F. Kadlec, Y. Yuzyuk, P. Simon, M. Pavel, et al., Ferroelectrics 176 (1996) 179.

[10] A.V. Belushkin, M. Bull, C. Carlile, S.G. Lushnikov, et al., Physics B 241-243 (1998) 484.

[11] A.I. Fedorov, Theory of Elastic Waves in Crystals, Plenum Press, New York, 1968.

[12] C. Hermann, Z. Kristallogr. 89 (1934) 32. 